\title{
PROFESORAS EN EL TERCER CICLO UNIVERSITARIO Y EN LOS POSTGRADOS SOBRE EEMM
}

\author{
Isabel de Torres Ramírez \\ Universidad de Granada
}

En los últimos años han proliferado los trabajos sobre la presencia de las mujeres en la Universidad española, no sólo como docentes e investigadoras, sino también como alumnas o, incluso, como miembros del $\mathrm{PAS}^{1}$. En este artículo se analizará tal presencia sólo dentro de la enseñanza y la investigación llevada a cabo en el tercer ciclo de los estudios universitarios y en los programas de postgrado, limitando el estudio al área de conocimiento "Estudios de las Mujeres" (en adelante EEMM) ${ }^{2}$. El objetivo que se persigue es estudiar la presencia de profesoras en los programas de doctorado, en la dirección y en los tribunales de tesis y también en los másters y programas expertos/as. Dado que éste es un campo muy cambiante, se procurará una información que ponga al día los datos que ya están recogidos en investigaciones anteriores, siempre que esto sea posible.

\footnotetext{
1 Véase, por ejemplo, Torres Ramírez, 2001. Pueden consultarse también, sobre alumnas y sobre profesoras en la universidad, Anguita y Robles, 1994; Alberdi, 2001 y García de León y García de Cortázar, 2001.

2 Hay algunas noticias que indican que en la Nomenclatura Internacional de la UNESCO para los campos de Ciencia y Tecnología ha sido o será en breve incluida dicha área de conocimiento; no se ha podido documentar tal inclusión, muy esperada por quienes cultivan los EEMM, por otra parte.
} 


\section{Mujeres en la enseñanza universitaria}

Según los últimos datos consignados en el sitio web del Instituto de la Mujer, referidos al curso 2002-2003 ${ }^{3}$, en dicho curso había en España 694.227 docentes, de los que 102.844 enseñaban en la universidad. Las mujeres representaban el 60,18\% del total y el 35,33\% de quienes en ese momento estaban presentes en la enseñanza universitaria. El cuadro siguiente recoge el porcentaje de mujeres presentes en la universidad, distribuidas por categorías académicas.

\section{Tabla 1}

Mujeres presentes en la universidad española, distribuidas por categorías académicas.

\begin{tabular}{|l|l|}
\hline Puesto & $\%$ \\
\hline Ayudantes & 48,75 \\
\hline Colaboradoras/es & 44,97 \\
\hline Profesoras/es titulares de escuelas universitarias & 40,97 \\
\hline Profesoras/es titulares de universidad & 35,36 \\
\hline Profesoras/es visitantes & 34,48 \\
\hline Asociadas/os & 34,41 \\
\hline catedráticas/os de escuelas universitarias & 31,90 \\
\hline catedráticas/os de universidad & 12,91 \\
\hline Eméritas/os & 8,56 \\
\hline Maestras/os de taller & 8,22 \\
\hline
\end{tabular}

Fuente: "La mujer en cifras". Estadísticas del Instituto de la Mujer.

\section{1. Mujeres en la docencia universitaria sobre EEMM}

En lo referente a la docencia relacionada con los EEMM en la universidad, la

\footnotetext{
${ }^{3}$ Cfr. http://www.mtas.es/mujer/mujeres/cifras/ (consultado el 17 de enero de 2006). Consultado igualmente el website del INE (Instituto Nacional de Estadística) el mismo día, me encuentro con que se indica allí que el "ultimo dato publicado" corresponde al dicho curso 2002-2003. No hay posibilidad, pues, de facilitar la información correspondiente a fecha más cercana, al menos utilizando datos publicados.
} 
casi totalidad de las personas que la llevan a cabo son mujeres. La información más reciente de la que se dispone a nivel nacional sobre la distribución de tales enseñanzas en los distintos niveles académicos es la que ofrece Cristina Segura en Universidad y Feminismo (II) (1999) ${ }^{4}$. Según puede deducirse de los datos que aporta la tabla 2, más del 55\% (55,4\%) de la enseñanza que se imparte sobre EEMM, Feministas y de Género tiene lugar dentro del tercer ciclo. A este elevado porcentaje hay que añadir un $22,1 \%$, que corresponde a las enseñanzas extracurriculares, dentro de las cuales, sin duda, se incluirán algunos másters y/o programas expertos. Sólo el 22,5\% (9,2\% asignaturas + $13,3 \%$ partes de programa) ocupan las enseñanzas regladas de los ciclos primero y segundo, en su mayor parte correspondientes a materias optativas o de libre configuración.

Tabla 2

Aportaciones de docencia (1992-95)

\begin{tabular}{|l|l|l|}
\hline Tipo Actividad & $\mathrm{N}^{\mathrm{o}}$ & $\%$ \\
\hline Asignatura & 55 & 9,2 \\
\hline Parte del Programa & 80 & 13,3 \\
\hline Doctorado & 333 & 55,4 \\
\hline Extracurricular & 133 & 22,1 \\
\hline
\end{tabular}

Fuente: Segura Graíño, 1999: 156

Teniendo en cuenta que los planes de estudios, en lo que a contenidos se refiere, no han cambiado en la última década; el número de asignaturas (55) y de partes de programa (80) impartidos no ha variado prácticamente, aunque habría que revisar los porcentajes, dada la cifra de nuevos programas de doctorado $\mathrm{y}$, sobre todo, de másters y programas expertos que se han creado en los años transcurridos del siglo XXI. Justamente es ésta la parcela que se va a revisar de forma pormenorizada,

\footnotetext{
${ }^{4}$ Una panorámica más actualizada, referida a Andalucía solamente, puede consultarse en Medina Guerra, 2004.
} 
centrando la información en los que están vigentes durante el curso 2005-2006, de acuerdo con los datos disponibles en la red ${ }^{5}$.

\section{Presencia de las Mujeres en la docencia universitaria de EEMM de tercer ciclo,} en la dirección de tesis y en los tribunales que las juzgan

Como ha quedado dicho, más de la mitad de la docencia impartida en la Universidad española que tiene como campo de conocimiento los EEMM corresponde al doctorado y son mayoritariamente mujeres quienes la imparten. Muy alta también es la autoría femenina de las tesis que se defienden es este campo. Abundante, aunque menos, la presencia de profesoras como directoras y codirectoras de las tesis que se generan y nada desdeñable el número de las que forman parte de los tribunales que las juzgan.

\subsection{Las profesoras en los Programas de doctorado en EEMM vigentes en el curso 2005-2006}

El número de programas sobre EEMM que en el curso 2005-2006 se están impartiendo asciende a 19 (en realidad 15, uno se imparte en tres universidades y 2 en dos), una cifra que casi coincide con la que recoge Teresa Ortiz para el curso 20022003, 16 cursos reseña (en realidad 13, uno impartido en cuatro universidades), aunque los programas no sean los mismos, hay algunos nuevos y otros que han desaparecido en 2005-2006 $6^{6}$. Las características que presentan tales cursos son las que se reflejan en la tabla 3 .

\footnotetext{
${ }^{5}$ La principal fuente de información utilizada han sido los website de las universidades españolas, consultados en enero de 2006.

${ }^{6}$ Cfr. Ortiz, Teresa. Op. Cit ( 2004), p. 143.
} 
Tabla 3

Programas de Doctorado sobre EEMM

vigentes en las universidades españolas durante el curso 2005-2006

\begin{tabular}{|c|c|c|c|c|c|c|}
\hline Univ. & $\begin{array}{l}\text { Título / } \\
\text { Denominación del } \\
\text { Programa }\end{array}$ & $\begin{array}{l}\text { Departamento/os Univ. } \\
\text { que participan }\end{array}$ & $\begin{array}{l}\text { Otras } \\
\text { Univ. }\end{array}$ & Carácter & $\begin{array}{l}\text { Sexo } \\
\text { Coor. }\end{array}$ & $\begin{array}{l}\text { Men- } \\
\text { ción de } \\
\text { Calidad }\end{array}$ \\
\hline UAL & \begin{tabular}{|l|} 
Estudios de Género. \\
Interuniversitario
\end{tabular} & Filologías Integradas & UJI & Interunivers. & $\mathrm{M}$ & No \\
\hline UCA & $\begin{array}{l}\text { Género, Identidad y } \\
\text { Ciudadanía }\end{array}$ & Filología Inglesa & UHU & Interunivers. & M & Sí \\
\hline & $\begin{array}{l}\text { La perspectiva feminista } \\
\text { como teoría crítica }\end{array}$ & $\begin{array}{l}\text { Economía Aplicada V / } \\
\text { Filosofía IV /Antropología } \\
\text { Social / Historia Moderna }\end{array}$ & & $\begin{array}{l}\text { Interfacultat. } \\
\text { Interdepart. }\end{array}$ & M & Sí \\
\hline UCM & $\begin{array}{l}\text { La perspectiva de } \\
\text { género en las ciencias } \\
\text { sociales }\end{array}$ & \begin{tabular}{|l|} 
Sociología I y IV / \\
Psicología Social / \\
Economía Aplicada V / \\
Antropología Social \\
\end{tabular} & & $\begin{array}{l}\text { Interdepart. } \\
\text { Interfacult. }\end{array}$ & $\mathrm{M} / \mathrm{V}$ & No \\
\hline & $\begin{array}{l}\text { Estudios de las mujeres } \\
\text { y de género* }\end{array}$ & $\begin{array}{l}\text { Instituto de Estudios de la } \\
\text { Mujer. }\end{array}$ & \begin{tabular}{|l|} 
USE \\
UMA \\
\end{tabular} & Interunivers. & $\mathrm{M}$ & No \\
\hline UGR & $\begin{array}{l}\text { Innovación en los } \\
\text { Cuidados de Salud de la } \\
\text { Mujer }\end{array}$ & Ginecología y Obstetricia & & Interdepart. & $\mathrm{V}$ & No \\
\hline UHU & $\begin{array}{l}\text { Género, Identidad y } \\
\text { Ciudadanía* }\end{array}$ & Filología Inglesa & UCA & Interunivers. & M & Sí \\
\hline UJA & $\begin{array}{l}\text { Mujeres, género y } \\
\text { estudios culturales* }\end{array}$ & $\begin{array}{l}\text { Lenguas y Culturas } \\
\text { Mediterráneas }\end{array}$ & ULL & \begin{tabular}{|l} 
Interdepart. \\
Interunivers.
\end{tabular} & M & No \\
\hline UJI & \begin{tabular}{|l|} 
Estudios e \\
investigaciones \\
interdisciplinarias en la \\
perspectiva de género
\end{tabular} & $\begin{array}{l}\text { Historia, Geografía y Arte } \\
\text { / Humanidades }\end{array}$ & UAL & Interunivers. & M & No \\
\hline ULL & $\begin{array}{l}\text { Mujeres, género y } \\
\text { estudios culturales }\end{array}$ & $\begin{array}{l}\text { Filología Clásica, } \\
\text { Francesa y Hispánica }\end{array}$ & UJA & Interunivers. & & No \\
\hline & $\begin{array}{l}\text { Relaciones de género, } \\
\text { sociedad y cultura en el } \\
\text { ámbito mediterráneo }\end{array}$ & Historia Contemporánea & & & $\mathrm{M}$ & No \\
\hline UMA & \begin{tabular}{|l|} 
Estudios de las Mujeres \\
y de Género
\end{tabular} & \begin{tabular}{|l|} 
Filología Española II / \\
Teoría de la Literatura
\end{tabular} & \begin{tabular}{|c|} 
UGR \\
USE \\
\end{tabular} & \begin{tabular}{|l|} 
Interdepart. \\
Interunivers.
\end{tabular} & $\mathrm{M}$ & No \\
\hline UOV & $\begin{array}{l}\text { Género y diversidad: } \\
\text { Metodologías, } \\
\text { investigación y } \\
\text { competencias }\end{array}$ & $\begin{array}{l}\text { Ciencia Política y de la } \\
\text { Administración / Filología } \\
\text { Alemana / Filologia } \\
\text { Inglesa / y otros }\end{array}$ & & Interdepart. & $\mathrm{M}$ & Sí \\
\hline UPO & Perspectiva feminista & Ciencias Sociales & & & $\mathrm{M} / \mathrm{V}$ & No \\
\hline
\end{tabular}




\begin{tabular}{|c|c|c|c|c|c|c|}
\hline & $\begin{array}{l}\text { del pensamiento } \\
\text { científico } \\
\text { contemporáneo. }\end{array}$ & & & & & \\
\hline & $\begin{array}{l}\text { La posición de la mujer } \\
\text { en el ordenamiento } \\
\text { jurídico }\end{array}$ & $\begin{array}{l}\text { Derecho Administrativo } \\
\text { Financiero y Procesal / } \\
\text { Derecho Privado / } \\
\text { Derecho Público General }\end{array}$ & & Interdepart. & M & No \\
\hline USAL & $\begin{array}{l}\text { Estudios } \\
\text { interdiscipinares de } \\
\text { género }\end{array}$ & $\begin{array}{l}\text { Geografía / Sociología y } \\
\text { comunicación / } \\
\text { Prehistoria, Historia } \\
\text { Antigua y Arqueología / y } \\
\text { otros }\end{array}$ & & Interdepart. & M & No \\
\hline & $\begin{array}{l}\text { Mujer, escrituras y } \\
\text { comunicación. } \\
\text { especialidad escritoras y } \\
\text { filología. esp literatura y } \\
\text { comunicación }\end{array}$ & Filología Alemana & & & & No \\
\hline USE & $\begin{array}{l}\text { Estudios de las mujeres } \\
\text { y de género }\end{array}$ & $\begin{array}{l}\text { Seminario de Estudios de } \\
\text { la Mujer./ Psicología } \\
\text { Experimental }\end{array}$ & $\begin{array}{l}\text { UGR } \\
\text { UMA }\end{array}$ & Interunivers. & M & No \\
\hline UVA & $\begin{array}{l}\text { Gènere, Subjetivitat, } \\
\text { Coneixement i Cultura }\end{array}$ & $\begin{array}{l}\text { Instituto Estudios de la } \\
\text { Mujer }\end{array}$ & & & $\mathrm{M}$ & No \\
\hline \multicolumn{7}{|c|}{ * Universidad que coordina el Programa } \\
\hline \multicolumn{7}{|c|}{$\begin{array}{l}\text { Acrónimos Universidades: UAL: Universidad de Alicante UCA: Universidad de Cádiz / UCM: } \\
\text { Universidad Complutense / UGR: Universidad de Granada / UHU: Universidad de Huelva / UJA: } \\
\text { Universidad de Jaén / UJI: Universidad Jaime I de Castellón / ULL: Universidad de Lleida / UMA: } \\
\text { Universidad de Málaga / UOV: Universidad de Oviedo / UPO: Universidad Pablo Olavide / USAL: } \\
\text { Universidad de Salamanca / USE: Universidad de Sevilla / UVA: Universidad de Valencia }\end{array}$} \\
\hline
\end{tabular}

De los datos que se ponen de manifiesto en la tabla precedente interesa subrayar que son varias las universidades en las que se imparte más de un programa integrable dentro de los EEMM, como sería el caso de la Autónoma de Madrid, Granada, Málaga y Salamanca. Salta también a la vista el carácter multidisciplinar de estos Estudios y el gran esfuerzo de coordinación que se está haciendo para lograr programas que muchas veces son interdeparmentales (7) y no pocas interuniversitarios (8). Es frecuente ver involucrados a los Institutos y Seminarios de EEMM en la organización de los mismos y, casi en el cien por cien de los casos, el programa está 
coordinado por una mujer. Aunque no se consigne el dato en la tabla, conviene destacar que el profesorado es en todos los casos mayoritariamente femenino. Esta presencia femenina mayoritaria se constata igualmente entre las coordinadoras adjuntas en el caso de los programas interuniversitarios, interfacultativos o interdepartamentales. Tres de ellos han conseguido la mención de calidad (de las universidades Complutense de Madrid, Huelva-Cádiz y Oviedo), con lo que tienen bastantes posibilidades de convertirse en postgrados.

Se ha prescindido aquí de mencionar los cursos de doctorado integrados en programas que no son específicamente de EEMM, aunque sean numerosos y pongan de manifiesto la transversalidad constatable de los dichos Estudios en las enseñanzas de tercer ciclo. Tales cursos suelen ser mayoritariamente impartidos por profesoras, según ponen de manifiesto los estudios precedentes ${ }^{7}$.

\subsection{Tesis: directoras y presencia en tribunales}

En un trabajo publicado recientemente por quienes esto escriben (Torres Ramírez y Torres Salina, 2005), se estudian las tesis doctorales sobre EEMM defendidas en las universidades españolas en los últimos veinticinco años para llegar a conocer el nivel de desarrollo de dichos Estudios. Entre los aspectos analizados se encuentran la autoría y la dirección de las tesis y la presencia de mujeres y varones en los tribunales que las juzgan. Prescindiendo de la primera de las cuestiones apuntadas -que tiene que ver también con la presencia de las mujeres en el tercer ciclo, pero referida a las alumnas y que no se estudiará aquí-, se ofrecen seguidamente los resultados referidos a la dirección de tesis y a los tribunales que las juzgan, a partir del corpus estudiado allí, que se extiende cronológicamente desde 1976-2002 y que usa como fuente la Base de Datos Teseo.

\footnotetext{
${ }^{7}$ Véase lo que se dice a tal respecto en el mencionado trabajo de Cristina Segura (1999), singularmente en las páginas 170 y 176.
} 


\subsubsection{Resultados que se derivan del análisis de la dirección de las tesis}

De las 412 tesis que integran el corpus analizado, un el $88 \%$ (361) tienen como autora a una mujer. No obstante, sólo el 43\% (177) han sido dirigidas por una profesora; 227 tienen como director a un varón, lo que supone un 55\% del total. Con los datos disponibles en Teseo, y a pesar de que se han hecho diversas gestiones que no siempre han dado resultado, de un $2 \%$ no se ha podido determinar la dirección. Aunque venga siendo algo habitual que, en términos absolutos, sean los profesores varones quienes dirigen el mayor número de las tesis que se presentan en las universidades españolas, debería buscarse alguna explicación al hecho de que los directores superen a las directoras también en este campo de conocimiento, sobre todo porque quienes cultivan los EEMM son preferentemente mujeres, como quedó dicho. Tomado globalmente, este dato pone de manifiesto que los varones ocupan en la escala académica la parte superior (hay más catedráticos que catedráticas, más directores que directoras de departamento y los investigadores principales de los proyectos son más frecuentemente varones) y que son ellos quienes mayoritariamente dirigen aquellas actividades que suponen mayor responsabilidad, que gozan de más prestigio y que proporcionan mayor poder, entre ellas la dirección de tesis doctorales. Otra interpretación posible sería considerar el hecho que se analiza como un síntoma de que los temas, nuevos objetos de estudio y nuevas perspectivas de investigación que proponen los EEMM van calando dentro de la universidad, empiezan a ser considerados científicamente y que ya no se marginan ni se limitan a las expertas, sino que otros profesores adoptan su metodología y se interesan por las cuestiones que plantean, dado que se trata de cuestiones ciertamente en alza y de metodologías nuevas y sugerentes. Incluso se podría pensar que, al menos en algunas ocasiones, son las doctorandas mayoritariamente representadas entre quienes cuentan con la dirección de un varónquienes proponen el tema a los posibles directores, por considerar que así tienen mayor posibilidad de obtener una beca o un mayor éxito profesional en el futuro, y que éstos 
Profesoras en el tercer ciclo universitario y en los postgrados sobre EEMM

aceptan, por razones diversas, aunque no sean especialistas en el enfoque ni en la temática propuestos. Muy interesante resulta comprobar la evolución que se produce en el número de tesis dirigidas por varones y por mujeres a lo largo de los años analizados, dado que quizá lo esperable sería que paulatinamente el número de directoras empezara a ser superior al de directores, a medida que las profesoras han ido consolidando su presencia y estatus académico y los EEMM se han institucionalizado.

\section{Gráfica 1}

Evolución de la dirección de tesis por años y sexo

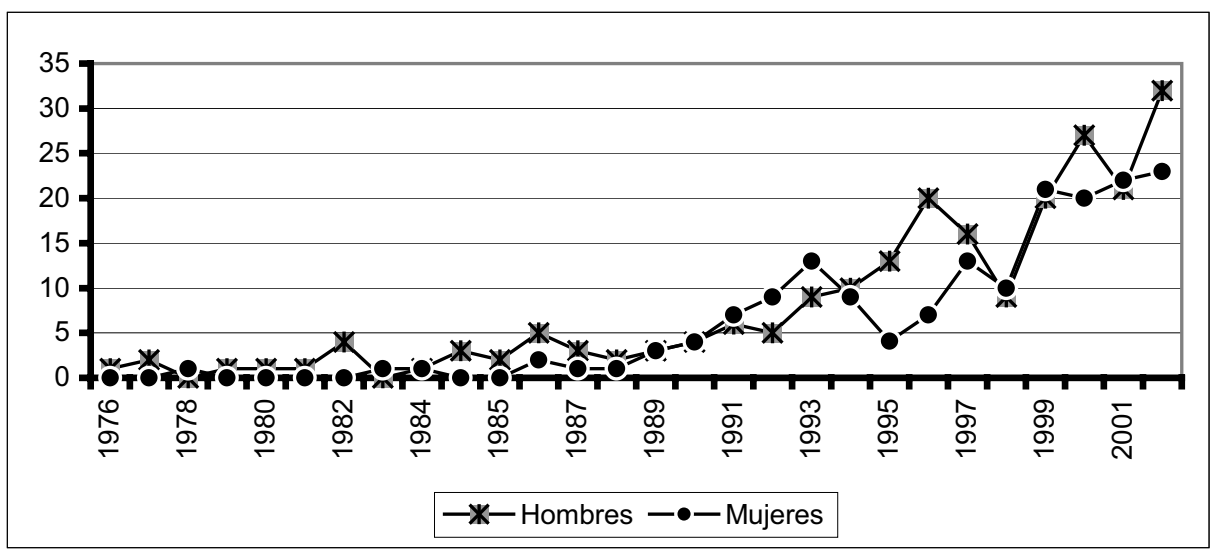

Sin embargo, la representación de la evolución pone en evidencia (gráfica 1) que, si bien las diferencias a favor de los varones son mayores al principio, nunca se llegan a igualar los valores, que -hasta 2002- siempre muestran la prevalencia masculina, con la excepción de los años comprendidos entre 1991 y 1993, donde puede apreciarse un pequeño repunte en las tesis dirigidas por mujeres, que nuevamente se sitúa por debajo de la escala masculina hasta el final, aunque en constante ascenso. 
Tabla 4

Codirecciones según número y sexo

\begin{tabular}{|l|l|r|}
\hline SEXO DIRECCIÓN & SEXO CODIRECCIÓN & $N^{\circ}$ \\
\hline Hombre & Hombre & 10 \\
\hline Hombre & Mujer & 14 \\
\hline Mujer & Hombre & 7 \\
\hline Mujer & Mujer & 3 \\
\hline
\end{tabular}

Quizá no esté de más puntualizar que al hacer el cómputo global de directoras y directores sólo se han contabilizado los que figuran como tales y no los que aparecen como codirectores /as, con lo que estos últimos no se reflejan. Sin embargo, en este caso la invisibilización afecta de manera idéntica a varones y mujeres, 17+17=34.

Tampoco debe despreciarse la observación de que, en algunas tesis codirigidas, la profesora que codirige no sea del Departamento en que se defiende la tesis, al que sí pertenece el director. Podría ser una prueba de que se reconoce su autoridad como experta y se solicita su colaboración necesaria, aunque quien figure como director sea un colega que, quizá, no quería o no sabía asumir el tema en solitario. La primera codirección se registra en una tesis defendida en 1998.

\subsubsection{Resultados en relación con la presencia de mujeres en los tribunales y comisiones que juzgan las tesis doctorales}

En relación con los tribunales, se constata en ellos una presencia mucho mayor de mujeres que de varones: 940 (71\%) mujeres, frente a 390 (29\%) varones). Este dato apunta a que quienes dirigen tesis doctorales y proponen las personas que han de juzgarlas son conscientes de que, en el campo de los EEMM, son las profesoras e investigadoras las más expertas, ya que son sus cultivadoras mayoritariamente.

Los datos anteriores se refieren al conjunto. En el porcentaje de varones y mujeres presentes en los tribunales puede observarse cómo, desde 1986, la presencia de mujeres es siempre muy superior a la de los varones, que nunca llega al $40 \%$, 
independientemente del sexo de la dirección y de la autoría, llegando tal diferencia a ser muy importante en 2002, año en que se aprecia una presencia femenina cercana al $80 \%$ (159 mujeres y 52 varones), contando con los datos que recoge la Teseo, que a veces omite esta información. Evidentemente, el porcentaje en cada caso se refiere al total de las tesis defendidas ese año, con lo que representa una cantidad muy diferente de presencias en 1976 (sólo se defendió 1 tesis) y 2002 (año en que se han seleccionado 55 tesis), todo multiplicado por los cinco miembros que integran las comisiones que juzgan dichos trabajos académicos.

\section{Gráfica 3}

Evolución de la presencia femenina y masculina en los tribunales de tesis

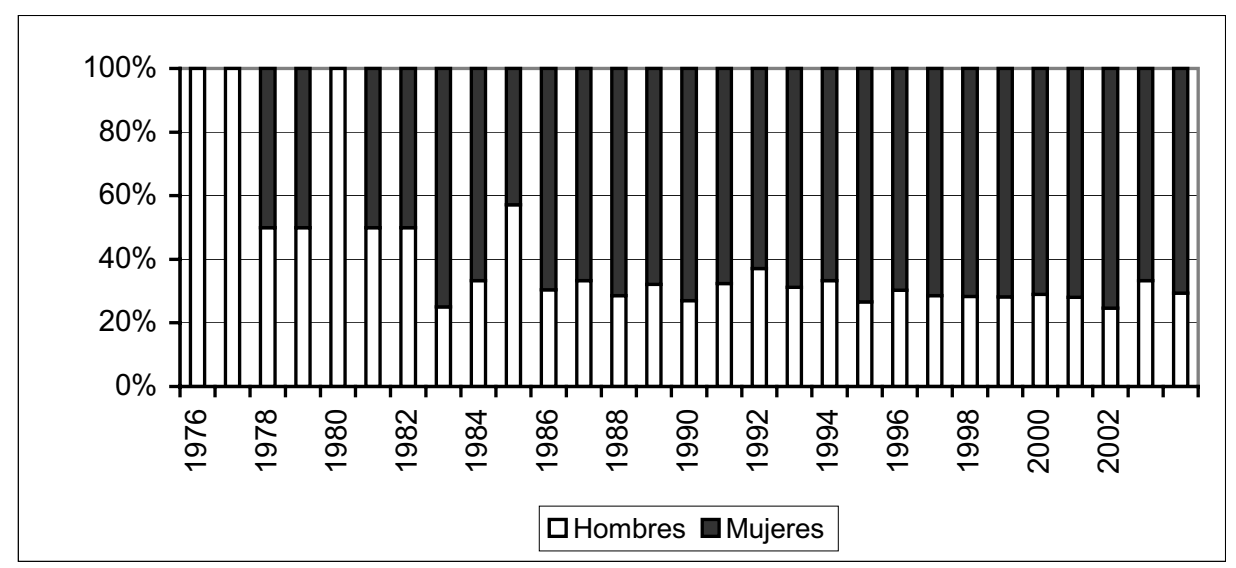

3. Mujeres en los cursos de postgrado (Máster, Experto/a y Especialista) en EEMM

Es en el campo de los llamados postgrados donde se ha dado un avance más espectacular en el número de cursos propuestos en los últimos tres años. Teresa Ortiz (2004: 144) registra en el curso 2002-2003 catorce postgrados -en su mayoría experta/o- que en el curso 2005-2006 han ascendido a treinta y uno), quince de ellos 
Másteres. La tabla 5 muestra de forma sistemática los postgrados vigentes en 20052006.

Tabla 5

Postgrados sobre EEMM vigentes en las universidades españolas durante el curso académico 2005-2006.

\begin{tabular}{|c|c|c|c|c|c|c|}
\hline UNIV. & ED & DENOMINACIÓN & CARÁCTER & ORGANIZA & $\begin{array}{l}\text { SEX. } \\
\text { COOR. }\end{array}$ & $\begin{array}{l}\text { DOCEN- } \\
\text { CIA }\end{array}$ \\
\hline UAL & & Violencia de género & Especialista & Servicio de Postgrado & $M$ & PRE \\
\hline UAM & & \begin{tabular}{|l} 
Estudios \\
Interdisciplinares de \\
Género \\
\end{tabular} & Máster & \begin{tabular}{|l} 
Instituto \\
Universitario de \\
Estudios de la Mujer \\
\end{tabular} & $\mathrm{M}$ & PRE \\
\hline \multirow[t]{2}{*}{ UBA } & & $\begin{array}{l}\text { Estudio de la diferencia } \\
\text { sexual }\end{array}$ & Máster & $\begin{array}{l}\text { Facultad de } \\
\text { Geografía e Historia } \\
\end{array}$ & $\mathrm{M}$ & O-L \\
\hline & & Estudios de las Mujeres & Máster & $\begin{array}{l}\text { Facultad de } \\
\text { Geografía e Historia }\end{array}$ & $\mathrm{M}$ & PRE \\
\hline \multirow[t]{6}{*}{ UCM } & & \begin{tabular}{|l} 
Estudios de la \\
Violencia de Género
\end{tabular} & Diploma & $\begin{array}{l}\text { Facultad CCs Polítca } \\
\text { y Sociología / } \\
\text { Instituto Mujer }\end{array}$ & $\mathrm{M}$ & PRE \\
\hline & & Feminismo y Género & Máster & \begin{tabular}{|l} 
Vicerrectorado de \\
Tercer Ciclo y \\
Formación Continua / \\
Instituto de \\
Investigaciones \\
Feministas \\
\end{tabular} & $M$ & PRE \\
\hline & & Género y Desarrollo & Máster & $\begin{array}{l}\text { Vicerrectorado de } \\
\text { Tercer Ciclo y } \\
\text { Formación Continua }\end{array}$ & V & PRE \\
\hline & $2^{\circ}$ & $\begin{array}{l}\text { Igualdad de Género: } \\
\text { Agentes y Políticas }\end{array}$ & Máster & Dpt de Sociología & $M$ & PRE \\
\hline & & $\begin{array}{l}\text { Políticas Europeas de } \\
\text { Género: Estudio } \\
\text { Comparativo. Experto. } \\
\text { Presencial }\end{array}$ & Experto/a & $\begin{array}{l}\text { Vicerrectorado de } \\
\text { Tercer Ciclo y } \\
\text { Formación Continua }\end{array}$ & $\mathrm{M}$ & PRE \\
\hline & $6^{\mathrm{a}}$ & $\begin{array}{l}\text { Sobre relaciones de } \\
\text { género y desarrollo }\end{array}$ & Diploma & $\begin{array}{l}\text { Facultad de Ciencias } \\
\text { políticas }\end{array}$ & $M$ & PRE \\
\hline UDC & & $\begin{array}{l}\text { Género y Políticas de } \\
\text { Igualdad }\end{array}$ & Máster & $\begin{array}{l}\text { Dpt de Socio. y } \\
\text { Ciencia Política de la } \\
\text { Admi. / Dpt } \\
\text { Pedagogía y }\end{array}$ & $M$ & PRE \\
\hline
\end{tabular}




\begin{tabular}{|c|c|c|c|c|c|c|}
\hline & & & & $\begin{array}{l}\text { Didáctica de Ciencias } \\
\text { experimentales }\end{array}$ & & \\
\hline \multirow[t]{2}{*}{ UDE } & & $\begin{array}{l}\text { Intervención en } \\
\text { violencia contra las } \\
\text { mujeres }\end{array}$ & Experto/a & $\begin{array}{l}\text { Facultad de Ciencias } \\
\text { políticas y Sociología }\end{array}$ & $\mathrm{M}$ & PRE \\
\hline & & \begin{tabular}{|l} 
Intervención en \\
violencia contra las \\
mujeres
\end{tabular} & Máster & $\begin{array}{l}\text { Facultad de Ciencias } \\
\text { políticas y Sociología }\end{array}$ & $M$ & PRE \\
\hline UGR & $5^{\mathrm{a}}$ & $\begin{array}{l}\text { Igualdad de género y } \\
\text { oportunidades }\end{array}$ & Experto/a & $\begin{array}{l}\text { Instituto de la Mujer } \\
\text { Vicerrectorado de } \\
\text { Postgrado y } \\
\text { Formación Continua }\end{array}$ & $\mathrm{M}$ & PRE \\
\hline \multirow[t]{2}{*}{ UJI } & & \begin{tabular}{|l} 
Curso de \\
Especialización en \\
Mainstreaming de \\
Género: alternativas \\
para la Igualdad
\end{tabular} & Especialista & $\begin{array}{l}\text { Centre d Estudis de } \\
\text { Postgrau i Formació } \\
\text { Continuada }\end{array}$ & $M$ & O-L \\
\hline & $3^{\circ}$ & $\begin{array}{l}\text { Curso de } \\
\text { Especialización en } \\
\text { Tratamiento No Sexista } \\
\text { de la Información: } \\
\text { Claves y Fundamentos }\end{array}$ & Experto/a & $\begin{array}{l}\text { Centre d Estudis de } \\
\text { Postgrau i Formació } \\
\text { Continuada }\end{array}$ & $\mathrm{M}$ & O-L \\
\hline ULA & $3^{\circ}$ & $\begin{array}{l}\text { Educación Sexual, } \\
\text { Terapia Sexual y } \\
\text { Género }\end{array}$ & Máster & Fundación Empresa & $\mathrm{V}$ & PRE \\
\hline ULL & & $\begin{array}{l}\text { Agentes de Igualdad de } \\
\text { Oportunidades para las } \\
\text { Mujeres; Acciones } \\
\text { Positivas en el Ámbito } \\
\text { Rural }\end{array}$ & Máster & $\begin{array}{l}\text { Seminari } \\
\text { Interdisciplina } \\
\text { d'Estudis de la Dona }\end{array}$ & $\mathrm{M}$ & O-L \\
\hline UMA & & $\begin{array}{l}\text { Género e Igualdad de } \\
\text { Oportunidades }\end{array}$ & Experto/a & Enseñanzas propias & $\mathrm{M}$ & PRE \\
\hline UNED & & $\begin{array}{l}\text { Agentes de Igualdad de } \\
\text { Oportunidades para las } \\
\text { Mujeres; Acciones } \\
\text { Positivas en el Marco } \\
\text { de la Cooperación } \\
\end{array}$ & Experto/a & $\begin{array}{l}\text { Virrectorado de } \\
\text { Educación } \\
\text { Permanente }\end{array}$ & $\mathrm{M}$ & DIS \\
\hline UPA & $3^{\circ}$ & $\begin{array}{l}\text { Igualdad de Mujeres y } \\
\text { Hombres }\end{array}$ & Máster & Emakunde & $\mathrm{M}$ & PRE \\
\hline URL & & $\begin{array}{l}\text { Cuerpo, Género y } \\
\text { Comunicación: La } \\
\text { construcción social del } \\
\text { cuerpo, la imagen y el } \\
\text { deseo en los medios } \\
\end{array}$ & Postgrado & $\begin{array}{l}\text { Facultad de } \\
\text { Comunicación } \\
\text { Blanquerna }\end{array}$ & $\mathrm{M}$ & PRE \\
\hline USC & $4^{\circ}$ & Educación, Género e & Máster & Instituto de Ciencias & $\mathrm{M}$ & PRE \\
\hline
\end{tabular}




\begin{tabular}{|c|c|c|c|c|c|c|}
\hline & & Igualdad & & $\begin{array}{l}\text { de la Educación / Dpt } \\
\text { Sociología / }\end{array}$ & & \\
\hline \multirow[t]{2}{*}{ USE } & & $\begin{array}{l}\text { Género e Igualdad de } \\
\text { Oportunidades }\end{array}$ & Experto/a & $\begin{array}{l}\text { Vicerrectorado de } \\
\text { Postgrado y } \\
\text { Doctorado } \\
\end{array}$ & $M$ & PRE \\
\hline & & $\begin{array}{l}\text { Participación y } \\
\text { Desarrollo desde una } \\
\text { Perspectiva de Género }\end{array}$ & Máster & \begin{tabular}{|l} 
Vicerrectorado de \\
Postgrado y \\
Doctorado
\end{tabular} & $M$ & PRE \\
\hline \multirow[t]{2}{*}{ UVA } & $7^{\circ}$ & Agentes de Igualdad & Máster & \begin{tabular}{|l|} 
Fundación \\
Universidad Empresa
\end{tabular} & $M$ & DIS \\
\hline & & $\begin{array}{l}\text { Agentes de Igualdad de } \\
\text { Oportunidades entre } \\
\text { Mujeres y Hombres }\end{array}$ & Especialista & $\begin{array}{l}\text { Servicio de Tercer } \\
\text { Ciclo / Fundación } \\
\text { General UVA }\end{array}$ & $M$ & PRE \\
\hline \multirow[t]{4}{*}{ UVI } & & $\begin{array}{l}\text { Especialista en Género } \\
\text { y Educación }\end{array}$ & Especialista & \begin{tabular}{|l|} 
Dpt de Socioloxía \\
Ciencia Política e da \\
admin.. e Filos e da \\
Cátedra Caixanova de \\
Estudios Feministas \\
\end{tabular} & $M$ & PRE \\
\hline & & $\begin{array}{l}\text { Especialista en Género } \\
\text { y Liderazgo }\end{array}$ & Especialista & \begin{tabular}{|l|} 
Dpt de Socioloxía \\
Ciencia Política e da \\
admin.. e Filos e da \\
Cátedra Caixanova de \\
Estudios Feministas \\
\end{tabular} & $\mathrm{M}$ & PRE \\
\hline & & $\begin{array}{l}\text { Especialista en Género } \\
\text { y Políticas de Igualdad }\end{array}$ & Especialista & \begin{tabular}{|l|} 
Dpt de Socioloxía \\
Ciencia Política e da \\
admin.. e Filos e da \\
Cátedra Caixanova de \\
Estudios Feministas
\end{tabular} & M & PRE \\
\hline & $2^{\circ}$ & $\begin{array}{l}\text { Género, Educación, } \\
\text { Políticas de Igualdad y } \\
\text { Liderazgo }\end{array}$ & Máster & \begin{tabular}{|l|} 
Dpt de Socioloxía \\
Ciencia Política e da \\
admin.. e Filos e da \\
Cátedra Caixanova de \\
Estudios Feministas
\end{tabular} & $M$ & PRE \\
\hline \multicolumn{7}{|c|}{$\begin{array}{l}\text { Acrónimo docencia: DIS: A distacia ; O-L: On-Line ; PRE: Presencial } \\
\text { Acrónimos Universidades: UAL: Universidad de Alicante / UAM: Universidad Autónoma de } \\
\text { Madrid / UBA: Universidad de Barcelona / /UCM: Universidad Complutense / UDC: } \\
\text { Universidad de la Coruña / UDE: Universidad de Deusto / UGR: Universidad de Granada / UJI: } \\
\text { Universidad Jaime I de Castellón / ULL: Universidad de Lleida / ULA: Universidad de la } \\
\text { Laguna / UMA: Universidad de Málaga / UNED: Universidad Nacional de Educación a } \\
\text { Distancia / UPA: Universidad del País Vasco / URL: Universidad Ramon jul / USC: Universidad } \\
\text { de Santiago de Compostela / USE: Universidad de Sevilla / UVA: Universidad de Valencia / } \\
\text { UVI: Universidad de Vigo }\end{array}$} \\
\hline
\end{tabular}


Además de los 15 másteres mencionados, se imparten en las universidades españolas públicas y privadas 7 cursos para obtener un título de experto/a, 6 para ser especialista y el resto (3) dan derecho a un Diploma de postgrado, sin especificación de título. No son pocas las universidades en las que existe un programa de doctorado que cuentan, además, con un curso de postgrado ( Complutense de Madrid, Granada, Lleida, Málaga, Sevilla y Valencia) y muchos de ellos se organizan o se animan desde un Instituto o Seminario de EEMM, cuyos miembros participan activamente entre el profesorado. La modalidad presencial es la más frecuente, hay algunos cursos on-line y dos a distancia. De la aceptación que tales cursos tienen da buena cuanta el hecho de que en nueve de ellos se celebren ediciones que van de la segunda a la séptima. Sólo dos de ellos tiene como director a un varón, lo que supone apenas el 7\%. Como en el caso de la dirección de los programas de doctorado, la presencia de mujeres directoras en los cursos de postgrado sobre género y mujeres es casi del 100\%, el 93,3 exactamente.

\section{Conclusiones}

Las conclusiones que se deducen de los datos analizados en relación con la presencia de mujeres en las Enseñanzas Superiores relacionadas con los EEMM en España podrían concretarse como sigue:

1. En lo que se refiere a la presencia de mujeres en los programas de doctorado, cuyo número ha aumentado en los últimos tres años de forma moderada, es frecuente que estos programas estén promovidos por los Institutos y Seminarios de estudios de las Mujeres, el profesorado es mayoritariamente femenino y la dirección corresponde en casi el cien por cien de los casos a una mujer. Esta presencia femenina mayoritaria se constata igualmente entre las coordinadoras adjuntas, en el caso de los programas interuniversitarios, interfacultativos o interdepartamentales.

2. Aunque la autoría de las 412 tesis pertenecientes al campo de conocimiento EEMM que se usan aquí como punto de referencia pertenezca mayoritariamente a 
doctorandas (361 de las 412, lo que viene a ratificar que son sobre todo mujeres quienes cultivan esta parcela académica), son sobre todo varones quienes dirigen las tesis analizadas: el 55\% del total. Este dato también parece ratificar un convencimiento generalizado y constatado estadísticamente en estudios recientes: las mujeres siguen estando infrarrepresentadas en los lugares donde se toman las decisiones dentro de la universidad y en las actividades de mayor responsabilidad y prestigio académico, tesis incluidas. Teniendo en cuenta que la tesis suele ser una puerta de integración profesional, no es aventurado pensar que quienes más tesis dirigen sean quienes tienen apoyos y recursos para situar a sus alumnos y alumnas, es decir, los profesores, más integrados en redes donde se les reconoce autoridad e influencia. Las codirecciones son escasas, 34 , y en 24 de ellas el primer director es también un varón.

3. En los tribunales que juzgan las tesis doctorales sobre EEMM es muy frecuente la presencia de doctoras, independientemente del sexo de la dirección y de la autoría. En 2002, más de $2 / 3$ de los miembros de dichas comisiones académicas fueron mujeres.

4. Es en el campo de los estudios de postgrado donde se ha producido un aumento más evidente, duplicándose los cursos en los últimos tres años. Tanto la docencia como la dirección y la codirección de estos cursos están en casi todos los casos en manos de mujeres, muchas veces vinculadas a los Institutos y Seminarios de Estudios de la Mujer que los promueven. 
Profesoras en el tercer ciclo universitario y en los postgrados sobre EEMM

\section{BIBLIOGRAFÍA}

ALBERDI, Inés (2001): “La carrera universitaria desde un punto de vista de género". En La mujer en el mundo académico. Madrid: L' Oreal España, pp. 125-147.

ANGUITA, Rocío y RoBLES, Victoria (1994): Informe sobre la presencia de las alumnas en la Universidad de Granada: 1983-1992. Granada: Universidad de Granada.

García de LeÓn María A. y GARcía de CoRTÁzAR, Marisa (2001): Las Académicas. (Profesorado universitario y género). Madrid: Instituto de la Mujer.

Ministerio de Trabajo y Asuntos Sociales. Instituto de la Mujer:

http://www.mtas.es/mujer/mujeres/cifras/.

Medina Guerra, Antonia María (2004): “Docencia e investigación en los estudios de las mujeres”. En Ruiz-Tagle, Ana María (coord.): Los Estudios de las Mujeres en las Universidades Andaluzas. Sevilla: Universidad de Sevilla, pp. 88-108.

OrTIZ GÓMEZ et al. (1999): Universidad y feminismo en España (II). Granada: Universidad de Granada.

Segura Graíño, Cristina (1999): "La docencia y los Estudios de las Mujeres, Feministas y de Género en la universidad". En ORTIZ GómEZ et al. Universidad y feminismo en España (II). Situación de los Estudios de las Mujeres en los años 90. Granada: Universidad de Granada, pp. 134-185.

TORRES RAMÍREZ, Isabel de (2001): “Mujeres en la Universidad de Granada. Un camino aún por recorrer”. En RUIZ RoDRÍGUEZ, Antonio. Memoria gráfica de la Universidad de Granada. Granada: Editorial Universidad de Granada, 2001, pp. 181-187.

TORRES RAMÍREZ, Isabel de y TORRES SALINAS, Daniel (2005): “Tesis doctorales sobre Estudios de las Mujeres en España (1976-2002). A propósito de un indicador definitivo en investigación". En Revista Española de Documentación Científica, vol. $28, n^{\circ} 4$, pp. $479-499$. 
